\title{
A search for peculiar objects in young open clusters
}

\section{New emission line and late spectral type stars in NGC $6871^{\star}$}

\author{
S. Bernabei ${ }^{1,2}$ and V. F. Polcaro ${ }^{3}$ \\ 1 Osservatorio Astronomico di Bologna, Via Ranzani 1, 40127 Bologna, Italy \\ 2 Dipartimento de Astrofísica, Universidad de La Laguna, Avda. Astrofisico F. Sánchez sn, 30071 La Laguna, \\ Spain \\ 3 Istituto di Astrofisica Spaziale, CNR, Area della Ricerca di Roma Tor Vergata, V. Fosso del Cavaliere snc, \\ 00133 Roma, Italy
}

Received 17 November 1999 / Accepted 9 November 2000

\begin{abstract}
We present the first results of a search in young open clusters for stars of very high initial mass, carried out by means of slitless spectroscopy. A deep $(R \simeq 17)$ survey of the open cluster NGC 6871 reveals many new objects of late spectral type, including an S star, as well as three new emission line stars, which are classified as Be stars. The already known Be star HD 227733 is surrounded by a previously undetected reflection nebula. The star VES 145, classified as an emission line object, is actually a late-type star, without any emission feature. We argue that VES 145 is a young RSG of a very high mass $\left(M_{0} \simeq 25 M_{\odot}\right)$ and a cluster member.
\end{abstract}

Key words. stars: early types - stars: emission-line, Be - stars: evolution - stars: individual (HD 227733, VES 145) - stars: late-type

\section{Introduction}

The evolution of very high mass stars $\left(M_{\text {initial }}>25 M_{\odot}\right)$ is intriguing and has been poorly understood for a long time. At present, although a number of powerful evolutionary codes have been developed (see Maeder 1996, for an exhaustive review), casting light on the general framework of the evolution of the high mass stars, many alternative scenarios are still possible. It is thus now necessary to provide more observational data allowing a better comparison between models and samples of stars that are homogeneous in age and initial chemical composition, but different in initial mass. In other words, it is necessary to find a class of objects playing the same role as that of the globular clusters in the evolutionary studies of the low mass stars.

The objects that can be used for an experimental test of evolutionary models of high and very high mass stars are, obviously, the young open clusters or associations where at least some objects with $M_{\text {initial }}>25 M_{\odot}$ (i.e. Of, LBV and WR stars, etc.) are present. However, this task is made difficult by the small number of stars of very

Send offprint requests to: V. F. Polcaro, e-mail: polcaro@saturn.ias.rm.cnr.it

* Based on data collected at $1.52 \mathrm{~m}$ "Cassini" Telescope of the Loiano Observing Station, Bologna Astronomical Observatory. high mass that are presently known, restricting the possibility of statistical studies. There are two different reasons for this. First, any reasonable IMF of a star association intrinsically involves a number of members decreasing with the stellar mass. Second, the high and very high mass stars evolve very rapidly; they are thus often located inside regions where the star formation processes are still active or are just ended and the residual interstellar matter produces a very strong local reddening. This fact implies that the high and very high mass stars, though intrinsically bright, are difficult to identify with a purely photometric survey. This problem increases when we are looking for the late evolutionary phases of stars with $M_{\text {initial }}>$ $25 M_{\odot}$ : actually, these objects, whose absolute luminosity is largely declined, are often embedded in dense circumstellar clouds, due to the strong mass loss during all evolutionary phases. The identification of high $\left(M_{0} \simeq 15-\right.$ $25 M_{\odot}$ ) mass stars is usually simpler. However, once again, their identification inside highly reddened regions on a purely photometric basis can be uncertain.

On the other hand, the identification of stars with very high $M_{\text {initial }}$ is unmistakable with a spectroscopic survey, because of their peculiarities. Also young high mass stars often show emission lines in some evolutionary stages (see e.g. Fabregat \& Torrejon 1999). In order to increase the 
statistics of this kind of objects we have thus started a survey of Northern young star associations based on slitless, objective prism-like spectroscopy, complemented by slit spectroscopy of the possible high and very high $M_{\text {initial }}$ candidates (emission line and late-type stars) and by CCD photometry.

As a first target, we selected the open cluster NGC 6871 because of a number of reasons. First of all, this object is well studied. We have thus the possibility to check our method on the literature data. Furthermore, the cluster includes many certain or possible very high $M_{\text {initial }}$ members: one WR star, many early supergiants and a number of objects with spectral peculiarities. On the other hand, the cluster is very dense and a number of filaments and dark clouds are visible in the field in the POSS plate. It is thus possible that more reddened and fainter objects remain undetected.

NCG 6871 belongs to the Cyg OB3 association. It is dominated by a a central core of massive stars, including WR 133 (HD 190918), classified as WN4.5+O9.5Ia (van der Hucht et al. 1981). Following Battinelli \& Capuzzo-Dolcetta (1991), NGC 6871 is located at 1649 pc from the Sun. Its age is $\leq 10 \mathrm{My}$, it has a dereddened integrated colour $(B-V)_{0}=-0.23$ and a turn-off colour $(B-V)_{\text {Tо }}=-0.25$. The galactic reddening in this direction is relatively high, with $E(B-V)=0.46$.

Massey et al. (1995) performed a deep photometric analysis of NGC 6871 , complemented by spectroscopy of a number of cluster members, in the framework of their studies on the initial mass function and massive stars evolution in the Milky Way. They estimated the mass of the highest mass star formed in the cluster as $M_{0} \simeq 40 M_{\odot}$ and the number of stars with $M_{0} \geq 10 M_{\odot}$ not less than 11 within a radius of $7.6 \mathrm{pc}$. The results of their survey is reported in a catalogue (known as MJD 95), where photometric data of 1955 stars and spectra of 11 members of NGC 6871 are given.

\section{Observations}

The observations of the NGC 6871 field have been performed in July 1999, using the Bologna Faint Object Spectrometer and Camera (BFOSC, Merighi et al. 1994) on the Cassegrain focus of the $1.52 \mathrm{~m}$ "Cassini" Telescope of the Bologna Astronomical Observatory, equipped with a $2048 \times 2048$ Loral CCD detector $(15 \mu \mathrm{m}$ pixel size, $13 \times 13 \operatorname{arcmin}^{2}$ FOV, 0.5 arcsec/pixel scale). We employed the slitless spectroscopy technique described by Polcaro \& Viotti (1997). This technique is based on the use of a low dispersion grism coupled with a broad-band filter giving an "objective prism-like" spectrogram of all the objects in the field, without a serious overlap of adjacent spectra. The use of a Johnson $R$ filter and of a 3500-9000 $\AA$, $\Delta \lambda=5.7 \AA$ grism, produces a $5600-8000 \AA$ bandpass imaging spectroscopy centered on $\mathrm{H} \alpha$, where emission line and late spectral type stars are easily identified. This slitless configuration implies a degraded spectral resolution that depends on seeing conditions, but greatly improves the $\mathrm{S} / \mathrm{N}$ ratio. The direct overlap of this spectral image and of the $R$ filter image allows the prompt and unambiguous correlation between the objects and their spectra.

Five fields surrounding the NGC 6871 core were taken with exposures times of $60 \mathrm{~s}$ for the Johnson $R$ filter photometry and $\sim 1200 \mathrm{~s}$ for the slitless spectroscopy in the range 5600-8000 $\AA$. A $R$ magnitude limit $\simeq 18$ was reached in the photometric images. The $\mathrm{S} / \mathrm{N}$ of the slitless spectra at $6000 \AA$ was $>100$ for early-type stars with $R=12$ mag. Better $\mathrm{S} / \mathrm{N}$ are of course obtained for late-type stars. Comparison between our data and the POSS digitized plates shows that no emission line objects brighter than $R \simeq 17$ remained undetected in our fields. Low resolution $(\Delta \lambda=5.7 \AA)$ slit spectra in the 3500 $9000 \AA$ range of the selected (emission line and late spectral type) stars were also obtained. Spectra of the standard star $\mathrm{BD}+28^{\circ} 4211$ (spectral type Op, $V=10.54$ ) were taken in the same nights with the same instrument set-up, for flux calibration.

In addition, a field covering the cluster core was observed with much lower exposure time in order to avoid saturation for the very bright stars WR 133 and $\mathrm{BD}+35^{\circ}$ 3953D. In the part of this field which does not overlap to the other ones, our completeness magnitude for emission line objects is much lower $(R \simeq 11)$. Table 1 gives the observation log and Fig. 1 shows the studied field. The positions of known and new emission line and late spectral type stars are also indicated.

Data were reduced and analysed using standard IRAF procedures and the resulting spectra were compared with various spectral atlases for spectral classification: Jaschek \& Jaschek (1987) and Jacoby et al. (1984), both covering all spectral types, Lennon et al. (1992) - Be stars -, Steele et al. (1999) - OB supergiants -, Barnbaum et al. (1996) - C stars -, Kirkpatrick et al. (1991) - late-type stars -, Keenan \& Boeshaar (1980) - S stars.

\section{Results}

All the previously known peculiar objects (WR 133 -WN4.5+O9.5Ia-, HD 227611 -B0II:pe-, HD 227733 -Beand VES 145) included in our fields were easily detected. We confirm their spectral classification reported in the SIMBAD data-base, except for the star VES 145 (see below). In addition to these objects, nine stars with emission lines or a late spectral type were newly discovered in our slitless spectroscopy and were further studied.

Tables 2 and 3 report their positions, $B$ and $V$ magnitudes and names from $S I M B A D$ when available, and our spectral classifications and $R$ magnitudes. Unfortunately, most nights were non photometric, thus no reliable magnitudes could be obtained from our data. However, we report in the tables a rough evaluations of the $R$ magnitude obtained from the mean of our photometric measurements and by integrating our flux calibrated spectra convolved with the Johnson $R$ passband.

These objects, provisionally named as BP, will be examined in detail in the following sections, together with a 


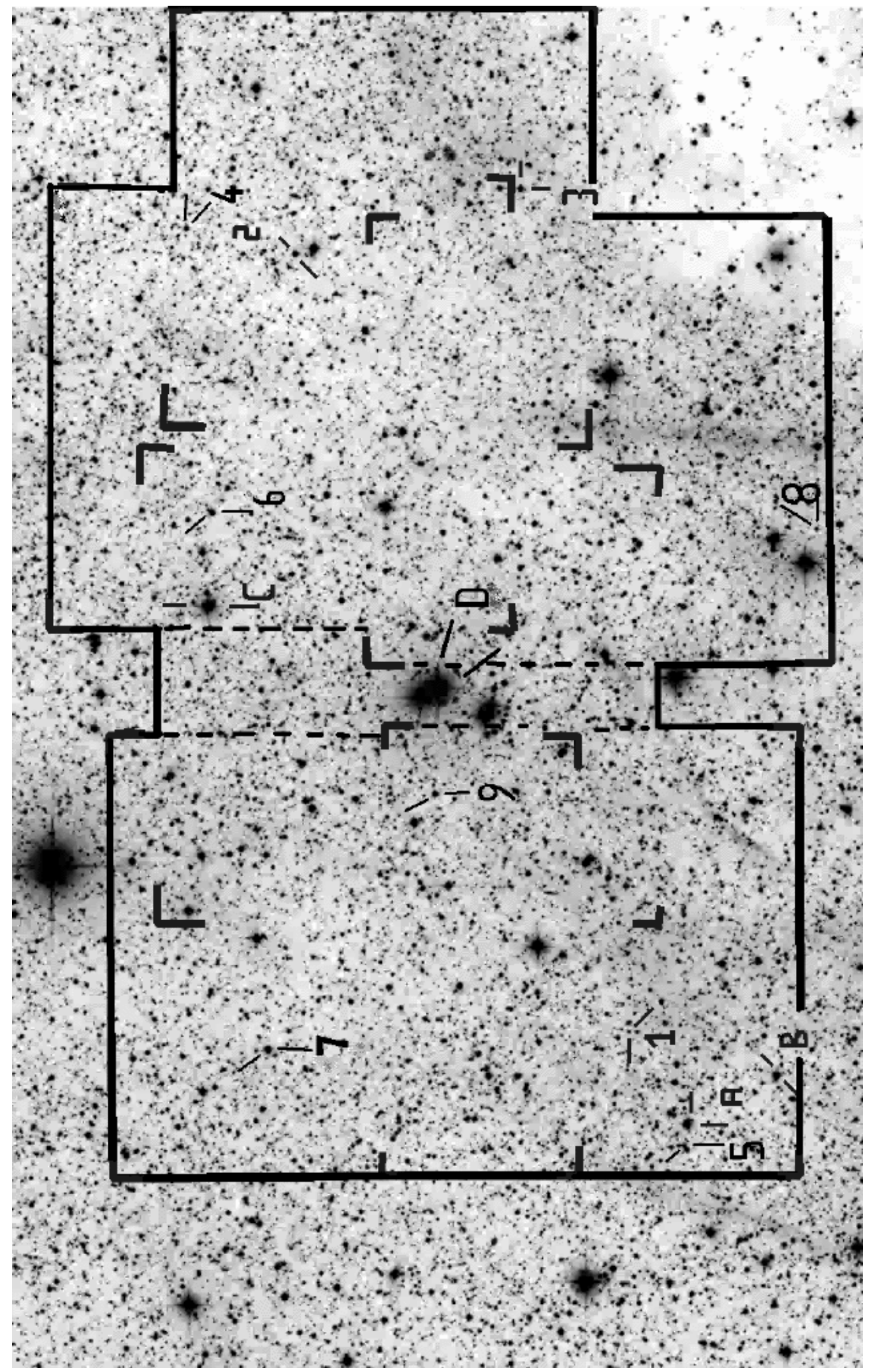

Fig. 1. The boundaries and corners of the six fields examined with the slitless spectroscopy are indicated on the NGC 6871 field from the digitized POSS plate; $\mathrm{N}$ is left and $\mathrm{W}$ is top. Dashed lines limit the area covered with only a short exposure. The positions of the new emission line stars $(1=\mathrm{BP} 1 ; 2=\mathrm{BP} 2 ; 3=\mathrm{BP} 3)$, of the new late-type stars $(4=\mathrm{BP} 4,5=\mathrm{BP} 5$, $6=\mathrm{BP} 6,7=\mathrm{BP} 7,8=\mathrm{BP} 8,9=\mathrm{BP} 9)$ and of the previously known peculiar objects $(\mathrm{A}=\mathrm{HD} 227733 ; \mathrm{B}=\mathrm{VES} 145$; $\mathrm{C}=\mathrm{HD} 227611, \mathrm{D}=\mathrm{WR} 133)$ are marked 
Table 1. Observation log of NGC 6871

\begin{tabular}{|c|c|c|c|c|c|}
\hline \multicolumn{6}{|l|}{ photometry } \\
\hline date & object & $\begin{array}{l}\text { exp. } \\
(\min )\end{array}$ & $\begin{array}{l}\text { UT beg } \\
\text { (hh:mm) }\end{array}$ & filter & $\begin{array}{l}\text { magnitude limit } \\
\quad(R \text { filter })\end{array}$ \\
\hline 1999 July 21 & Field 1 & 1.0 & $02: 28$ & $R$ & 18 \\
\hline 1999 July 21 & Field 2 & 1.0 & $20: 48$ & $R$ & 18 \\
\hline 1999 July 21 & Field 3 & 1.0 & $22: 02$ & $R$ & 18 \\
\hline 1999 July 21 & Field 4 & 1.0 & $22: 47$ & $R$ & 18 \\
\hline 1999 July 21 & Field 5 & 0.1 & $23: 30$ & $R$ & 12 \\
\hline 1999 July 21 & Field 6 & 1.0 & $23: 49$ & $R$ & 18 \\
\hline \multicolumn{6}{|c|}{ slitless spectroscopy } \\
\hline date & object & $\begin{array}{l}\text { exp. } \\
(\min )\end{array}$ & $\begin{array}{l}\text { UT beg } \\
\text { (hh:mm) }\end{array}$ & $\begin{array}{l}\text { sp. range } \\
(\AA)\end{array}$ & $\begin{array}{l}\text { res. } \\
(\AA)\end{array}$ \\
\hline 1999 July 21 & Field 1 & 15 & 02:30 & $5600-8000$ & 20 \\
\hline 1999 July 21 & Field 1 & 20 & $02: 46$ & $5600-8000$ & 20 \\
\hline 1999 July 21 & Field 2 & 20 & $20: 58$ & $5600-8000$ & 20 \\
\hline 1999 July 21 & Field 3 & 20 & $22: 08$ & $5600-8000$ & 20 \\
\hline 1999 July 21 & Field 4 & 20 & $22: 53$ & $5600-8000$ & 20 \\
\hline 1999 July 21 & Field 5 & 0.3 & $23: 47$ & $5600-8000$ & 20 \\
\hline 1999 July 21 & Field 6 & 20 & $23: 58$ & $5600-8000$ & 20 \\
\hline \multicolumn{6}{|c|}{ slit spectroscopy } \\
\hline date & object & $\begin{array}{l}\text { exp. } \\
(\min )\end{array}$ & $\begin{array}{l}\text { UT beg } \\
\text { (hh:mm) }\end{array}$ & $\begin{array}{l}\text { sp. range } \\
(\AA)\end{array}$ & $\begin{array}{l}\text { res. } \\
(\AA)\end{array}$ \\
\hline 1999 July 22 & $\mathrm{BP} 1$ & 30 & $20: 15$ & $3500-9000$ & 5.7 \\
\hline 1999 July 23 & BP $5+$ HD 227733 & 30 & $02: 27$ & $3500-9000$ & 5.7 \\
\hline 1999 July 23 & $\mathrm{BD}+28^{\circ} 4211$ & 10 & 03:05 & $3500-9000$ & 5.7 \\
\hline 1999 July 23 & VES 145 & 20 & $20: 20$ & $3500-9000$ & 5.7 \\
\hline 1999 July 23 & BP 2 & 30 & $21: 15$ & $3500-9000$ & 5.7 \\
\hline 1999 July 23 & $\mathrm{BP} 4$ & 30 & $21: 55$ & $3500-9000$ & 5.7 \\
\hline 1996 July 24 & $\mathrm{BD}+28^{\circ} 4211$ & 20 & 01:06 & $3500-9000$ & 5.7 \\
\hline 1999 July 25 & BP 3 & 30 & $21: 25$ & $3500-9000$ & 5.7 \\
\hline 1996 July 25 & $\mathrm{BD}+28^{\circ} 4211$ & 20 & $01: 36$ & $3500-9000$ & 5.7 \\
\hline
\end{tabular}

Table 2. New emission line objects in NGC 6871

\begin{tabular}{cccccccc}
\hline name & alternative names & spectral type & \multirow{2}{*}{ RA (2000) } & Decl. (2000) & $B$ & $V$ & $R$ \\
\hline BP 1 & & B:e & $20 \mathrm{~h} 4 \mathrm{~m} 47.5 \mathrm{~s}$ & $3541^{\prime} 45^{\prime \prime}$ &.. &.. & $15.4 \pm 0.5$ \\
BP 2 & (MJD 95) J200254.07+3551214 & B5Ve & $20 \mathrm{~h} 04 \mathrm{~m} 54.1 \mathrm{~s}$ & $3551^{\prime} 20^{\prime \prime}$ & 11.85 & 11.98 & $12.4 \pm 0.5$ \\
BP 3 & (MJD 95) J2004450+354441 & B8Ve & $20 \mathrm{~h} 05 \mathrm{~m} 31 \mathrm{~s}$ & $3553^{\prime} 53^{\prime \prime}$ & 12.66 & 12.75 & $12.1 \pm 0.5$ \\
\hline
\end{tabular}

number of new results on the previously known peculiar objects.

\subsection{Emission line objects}

$B P 1$

No previous name or position is reported in $S I M B A D$ for this star.
It is the faintest new object of our survey, thus a precise spectral classification is extremely difficult (see Fig. 2). However, He I absorption lines $(\lambda \lambda 6678,7065,7281 \AA)$ are clearly visible in the red part of BP 1 spectrum, where the signal to noise ratio becomes $\geq 10$ (see Fig. 3 ). Although the presence of these lines is not in itself a final proof of a $\mathrm{B}$ spectral type, being this ion also present in late $\mathrm{O}$ type stars (e.g. Jaschek \& Jaschek 1987), the absence or very weak presence of the He II lines, that are strong in O type 

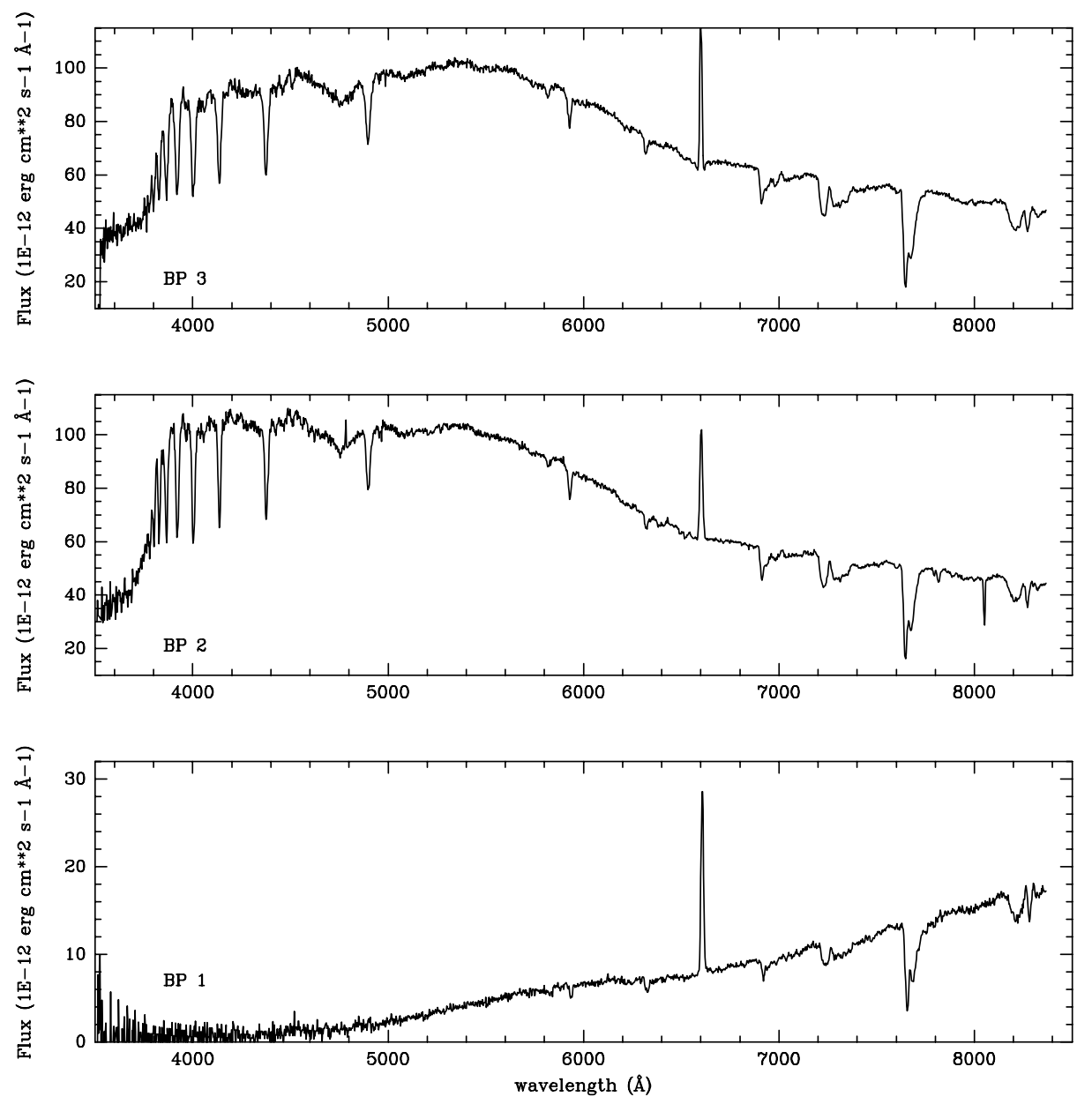

Fig. 2. The spectra of BP 1, BP 2 and BP 3 in the 3500-9000 $\AA$ range

stars, suggests that BP 1 is a heavily reddened early Be star.

Assuming this spectral type and a temperature range of $15000 \mathrm{~K}$ to $30000 \mathrm{~K}$, an $E(B-V)=1.2 \pm 0.2$ can be argued from the fit of the star continuum. Such a high value of the reddening implies that this star is deeply embedded in a local cloud. From the $\mathrm{H} \alpha$ equivalent width (= $42.4 \AA$ ), we can evaluate a circumstellar component of the reddening corresponding to a $E_{\mathrm{cs}}=0.07$ (Fabregat \& Reglero 1990). Actually, Massey et al. (1995) found local reddening values up to $E(B-V)=1.1$ in NGC 6871 . Although we cannot completely rule out the possibility that it could be a background star, the high local interstellar reddening of NGC 6871 seems to favour the first explanation.

\section{$B P 2$ and $B P 3$}

Both stars are included in the MJD 95 catalogue, without spectral classifications. Both stars show a clear B spectrum with strong emission in $\mathrm{H} \alpha$ (see Fig. 2). The wide Balmer lines suggest a luminosity class V. In order to get an accurate spectral classification, we compared their spectra with the Steele et al. (1999) catalogue of Be stars and with the general recepees of Jaschek \& Jaschek (1987). The shape of the blend at $\sim 4460-4500 \AA$, mostly due to the He I $4471 \AA$ and Mg II $4480 \AA$ lines, suggests that these two lines have comparable strength in both stars, wich indicates a spectral type later than B4. However, the BP 2 spectrum shows also weak highly ionised metal lines, and particularly Si III $\lambda \lambda 4552,4568 \AA$, that disappear at the spectral type B5. These lines are lacking in the BP 3 spectrum. Because of this, and of the $\mathrm{H} \gamma$ equivalent widths (6.4 $\AA$ and $6.9 \AA$ in $\mathrm{BP} 2$ and BP 3 , respectively) we classify BP 2 as B5Ve and BP 3 as B8Ve. The $\mathrm{H} \beta$ equivalent widths of the two stars $(4.4 \AA$ and $7.9 \AA$, respectively) are slightly lower than the figures quoted by Jaschek \& Jaschek (1987) for these spectral types, most probably because of the presence of an emission component undetected at our resolution.

The reddenings, obtained by fitting the flux calibrated spectra of BP 2 and BP 3 with the Kurucz (1979) models corresponding to the effective temperature of a B5V and B8V star $(15400 \mathrm{~K}$ and $11900 \mathrm{~K}$ respectively, SchmidtKaler 1982) are $0.7 \pm 0.1$ for BP 2 and $0.5 \pm 0.1$ for BP 3 . These are both compatible with the $E(B-V)$ range of NGC 6871 found by Massey et al. (1995). Part of this reddening is due to circumstellar material; however, following Eq. (1) of Fabregat \& Reglero (1990), from the measured values of the $\mathrm{H} \alpha$ equivalent widths $(-11.8 \AA$ and $-12.7 \AA$ for BP 2 and BP 3, respectively) the circumstellar 


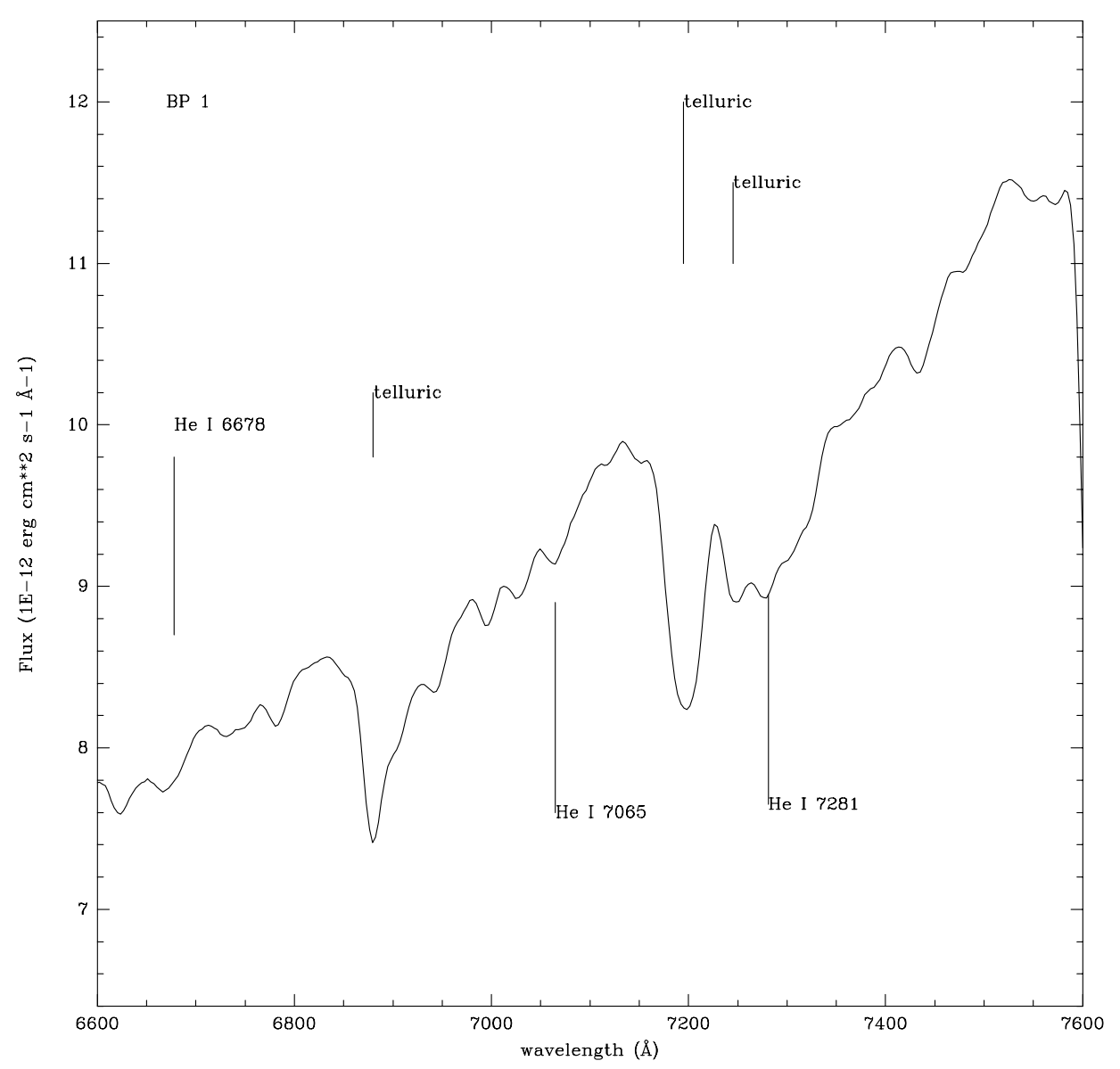

Fig. 3. The spectrum of BP 1 in the $6600-7600 \AA$ range. He I features are shown

reddening is only $\sim 0.02 \mathrm{mag}$ in both cases. We can thus argue that BP 3 is located on the boundary of NGC 6871 , while BP 2 is embedded in an intracluster cloud. This fact is also supported by the depth of the interstellar bands in the BP 2 spectrum.

The positions of BP 2 and BP 3, in the $\log T_{\text {eff }}, M_{\text {bol }}$ plane, evaluated using the figures given by Schmidt-Kaler (1982) for B5V and B8V stars, fit the the Schaller et al. (1992) tracks for $M_{0} \simeq 7 M_{\odot}$ for $\mathrm{BP} 2$ and $\sim 4 M_{\odot}$ for BP 3 (see Fig. 5), not far from the TAMS, as found in the case of many Be stars (e.g. Fabregat \& Torrejon 1999).

Their position in the cluster HR diagram reported by Massey et al. (1995) is not unusual for Be stars (e.g., Caloi \& Cassatella 1998).

\section{HD 227733}

This object was partially in the slit of the BP 5 spectrum. We thus obtained also a spectrum of this star, although underexposed. We can confirm the Be spectral classification reported in $S I M B A D$. However, it is interesting to notice that the cross section of the spectrogram clearly shows a diffuse emission surrounding this star (see Fig. 6), that is not reported in literature, despite the emission is also visible in the POSS plate. The spectrum of this nebulosity (though very faint) clearly shows its nature of circumstellar reflection nebula.

\subsection{Late spectral type objects}

Inside NGC 6871, we discovered six late-type stars, named BP 4, BP 5, BP 6, BP 7, BP 8 and BP 9. Furthermore, we discovered that also the cluster member known as VES 145 is actually a late-type star. These objects will be discussed in detail in the following.

\section{VES 145}

This object is reported in $S I M B A D$ as an emission line object and its name comes from the Vatican Emissionline Survey, but its spectrum is clearly that of a latetype (M0I) supergiant, with no emissions (see Fig. 7). On the other hand, it is well know that the VES catalogue contains a few late spectral type objects where the band heads were misunderstood as emission lines, due to the poor spectral resolution of the objective prism employed (MacConnell \& Coyne 1983). Of course, we cannot rule out also the possibility that, at the time of the Vatican survey, $\mathrm{H} \alpha$ emission was actually present, as it happens in both single and binary late-type stars.

We have computed the absolute magnitude of VES 145, on the basis of the distance and reddening of NGC 6871 given by Battinelli \& Capuzzo-Dolcetta (1991). The calculated value, $V_{0}=-2.1$, corresponds to a luminosity class I. Given the star temperature derived from 

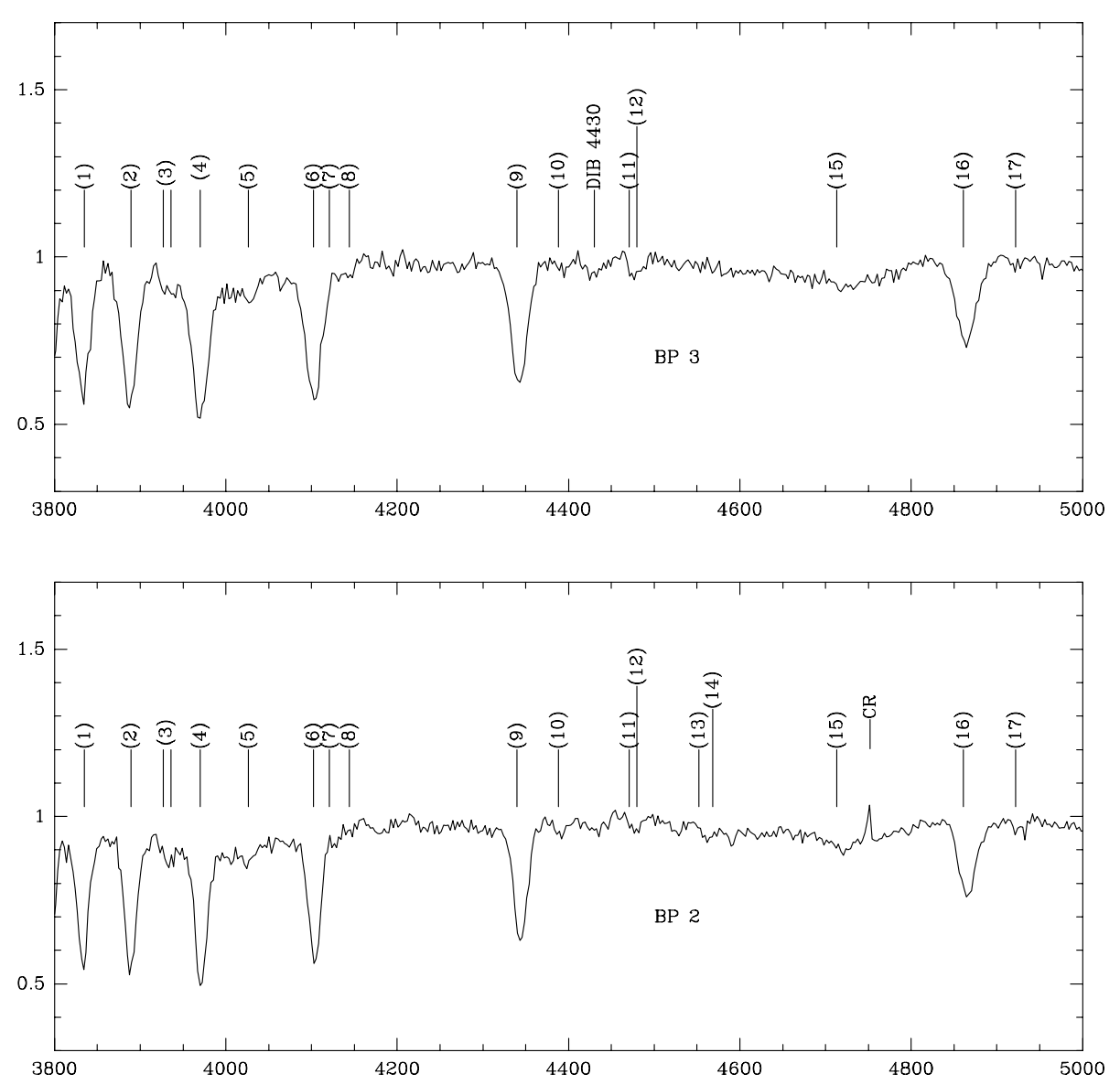

Fig. 4. The normalized spectra of BP 2 and BP 3 in the 3800-5000 A range. The features used for spectral classification are: $1=\mathrm{H} 93835,2=\mathrm{H} 8$ 3889, $3=$ He I 3926-36, $4=\mathrm{H} \epsilon 3970,5=$ He I 4026, $6=\mathrm{H} \delta$ 4102, $7=$ He I 4121, $8=\mathrm{He}$ I 4144, $9=\mathrm{H} \gamma$ 4340, $10=\mathrm{He}$ I 4388, $11=\mathrm{He}$ I 4471, $12=\mathrm{Mg}$ II 4480, $13=\mathrm{Si}$ III 4552, $14=\mathrm{Si}$ III 4568, $15=\mathrm{He}$ I 4713, $16=\mathrm{H} \beta 4861$, $17=$ He I 4922

Table 3. Late spectral type stars in NGC 6871

\begin{tabular}{|c|c|c|c|c|c|c|c|}
\hline name & alternative names & spectral type & RA (2000) & Decl. (2000) & $B$ & $V$ & $R$ \\
\hline $\mathrm{BP} 4$ & & M3.5III & $20 \mathrm{~h} 04 \mathrm{~m} 50 \mathrm{~s}$ & $3554^{\prime} 32^{\prime \prime}$ & & & $12.4 \pm 0.5$ \\
\hline $\mathrm{BP} 5$ & & $\mathrm{~S} 6 / 6$ & $20 \mathrm{~h} 06 \mathrm{~m} 57 \mathrm{~s}$ & $3540^{\prime} 04^{\prime \prime}$ & & & $13.6 \pm 0.5$ \\
\hline BP 6 & (MJD 95) J200531.06+355401 & M: & $20 \mathrm{~h} 05 \mathrm{~m} 31 \mathrm{~s}$ & $3553^{\prime} 53^{\prime \prime}$ & 12.36 & 12.19 & $12.1 \pm 0.5$ \\
\hline BP 7 & & M: & $20 \mathrm{~h} 06 \mathrm{~m} 50 \mathrm{~s}$ & $3552^{\prime} 23^{\prime \prime}$ & & & $11.9 \pm 0.5$ \\
\hline $\mathrm{BP} 8$ & & M: & $20 \mathrm{~h} 05 \mathrm{~m} 34 \mathrm{~s}$ & $3536^{\prime} 06^{\prime \prime}$ & & & $12.5 \pm 0.5$ \\
\hline $\mathrm{BP} 9$ & & M: & $20 \mathrm{~h} 06 \mathrm{~m} 13 \mathrm{~s}$ & $3547^{\prime} 26^{\prime \prime}$ & & & $12.3 \pm 0.5$ \\
\hline VES 145 & & MOI & $20 \mathrm{~h} 06 \mathrm{~m} 26 \mathrm{~s}$ & $3539^{\prime} 01^{\prime \prime}$ & 12.4 & 10.3 & $10.5 \pm 0.5$ \\
\hline
\end{tabular}

its spectral type and luminosity class (3650 K, Zombeck 1990) the representative point of VES 145 fits fairly well the Schaller et al. (1992) $25 M_{\odot}$ track in the HR diagram of NGC 6871. We thus conclude that this star actually is a very high $M_{\text {initial }}$ member of NGC 6871 .

$B P 4$

No previous name or position are reported in SIMBAD for this star. We obtained slit spectra of BP 4, that we classified as M3.5 (see Fig. 8), by comparison with the corresponding standard stars of the Jacoby et al. (1984),
Jaschek \& Jaschek (1987) and Kirkpatrick et al. (1991) catalogues. The weakness of CN $7878 \AA$ band and 84988542-8662 $\AA$ Ca II triplet suggest a luminosity class III. The object is thus much older than the cluster age and possibly is not a member of NGC 6871. However, its measured $R$ magnitude is compatible with the cluster distance.

$B P 5$

No previous name or position are reported in SIMBAD for this star. This object shows a clear S spectrum, very similar to that of R Cyg (see Jaschek \& Jashek 1987, p. 369). 


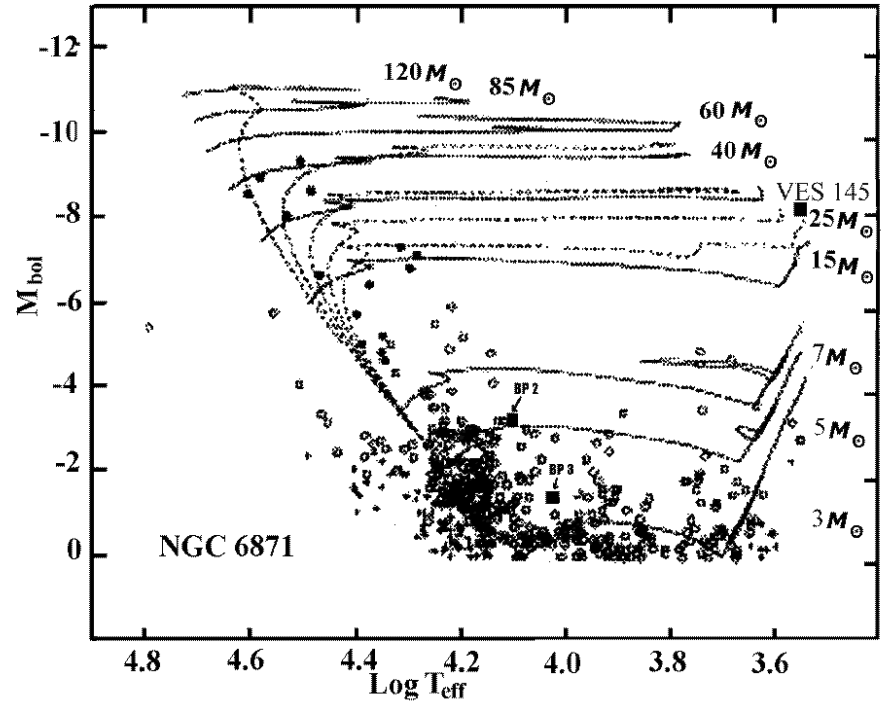

Fig. 5. The HR diagram of NGC 6871 reported by Massey et al. (1995). Schaller et al. (1992) tracks and the representative points of BP 2, BP 3 and VES 145 (filled squares) are also shown

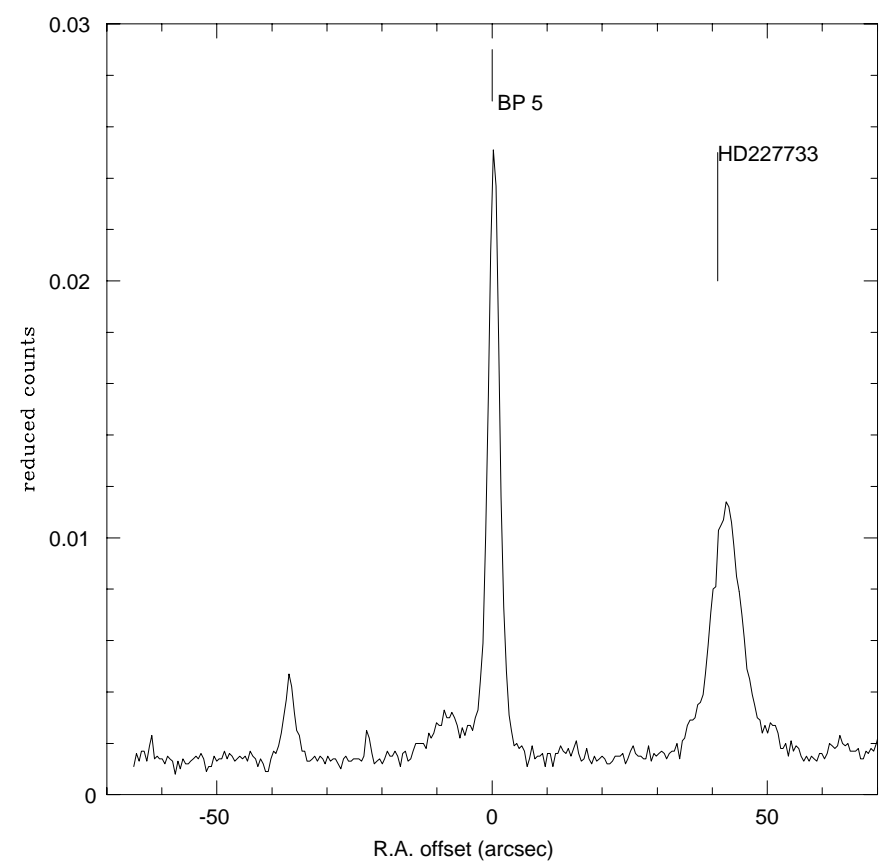

Fig. 6. E-W cross section of the BP 5 and HD 227733 spectrogram at $\lambda=6000 \AA$. The diffuse emission around HD 227733 is clearly visible

All the typical features of this class of stars are clearly visible: the $\mathrm{ZrO}$ band series is very strong, while all the $\mathrm{TiO}$ bands $(4954,4626,4584,4422 \AA)$ are weak and the LaO bands (7404, 7900), C2 and YO bands are present (see Fig. 9). Because of these features, we propose the values $\beta=6$ and $\alpha=6$, for the $\mathrm{S}$ stars spectral classification parameters introduced by Keenan \& Boeshaar (1980). Unfortunately, it is very hard to estimate the luminosity class of this kind of objects and we can not state at present whether BP 5 is a member of NGC 6871. Furthermore, it is also extremely difficult to perform reliable measures of the interstellar absorption features on such a complex spectrum. We can argue that, if BP 5 is a cluster member, it must be a supergiant of very high initial mass, but, to our knowledge, no evolutionary theory foresees an $\mathrm{S}$ star phase for this kind of objects. On the other hand, Jorgensen (1985) demonstrated that there are no statistical reasons to believe that any of the known S star can be regarded as a cluster member only because it lies in the same line of sight.

\section{$B P 6, B P \%, B P 8, B P 9$}

Only slitless spectra of BP $6, \mathrm{BP} 7, \mathrm{BP} 8$ and BP 9 were obtained (see Fig. 10). Due to the low spectral resolution, their exact spectral types are still poorly defined, though all their spectra are certainly of M type, and their luminosity classes are unknown. We cannot thus argue whether they are also members of the cluster.

We want only to notice that the $B$ and $V$ magnitudes reported by Massey et al. (1995) for BP 6 (=MJD 95 J200531.06+355401), the only object of this group included in the MJD 95 catalogue, do not fit the energy distribution of an $\mathrm{M}$ star. This object thus needs to be further investigated in more detail.

\section{Conclusion}

Our survey shows that no other WR or Oe stars brighter than $R \simeq 17$, apart from the previously known ones, are present inside the field studied. This result is not surprising due to the brightness and peculiar spectra of these objects and the large number of previous studies on NGC 6871. The spectral range of our slitless spectroscopic survey reduces the possibility to easily identify Of stars, but, because of the above reasons, we can suppose that all these objects are also already known.

On the other hand, we found at least one new RSG and 3 new Be stars, rising to 6 the total number of emission line stars (considering that VES 145 is not presently an object of this kind) known in this field.

The method that we have used, appears thus quite effective to discover previously unrecorded emission line and late spectral type stars in young open clusters. However, it will have to be improved by using different combinations of grisms and photometric filters in order to allow an effective detection of all the very high $M_{\text {initial }}$ stars candidates.

Acknowledgements. We thank Drs R. Viotti and V. Caloi (CNR-IAS) for useful discussions and the unknown referee for very valuable suggestions and comments. This work has made use of the SIMBAD database at the CDS (Strasbourg). Detailed finding charts and the reduced spectrograms of the objects listed in Tables 2 and 3 are available as computer files upon request to the authors at the address: polcaro@saturn.ias.rm.cnr.it 

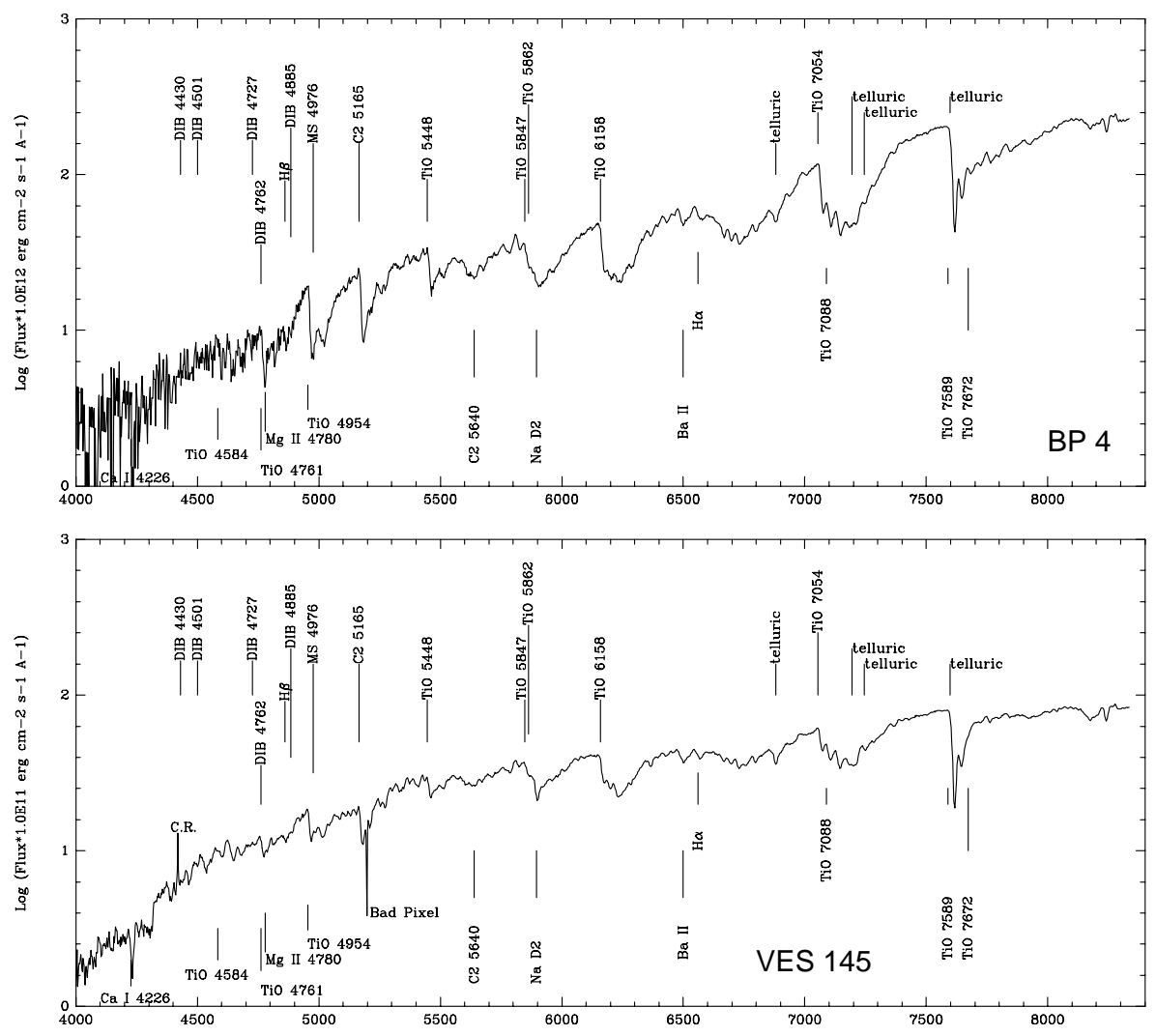

Fig. 7. The flux calibrated spectrum of VES 145 and BP 4 in the 3800-8400 $\AA$ range. Lines and bands used for spectral classification are shown

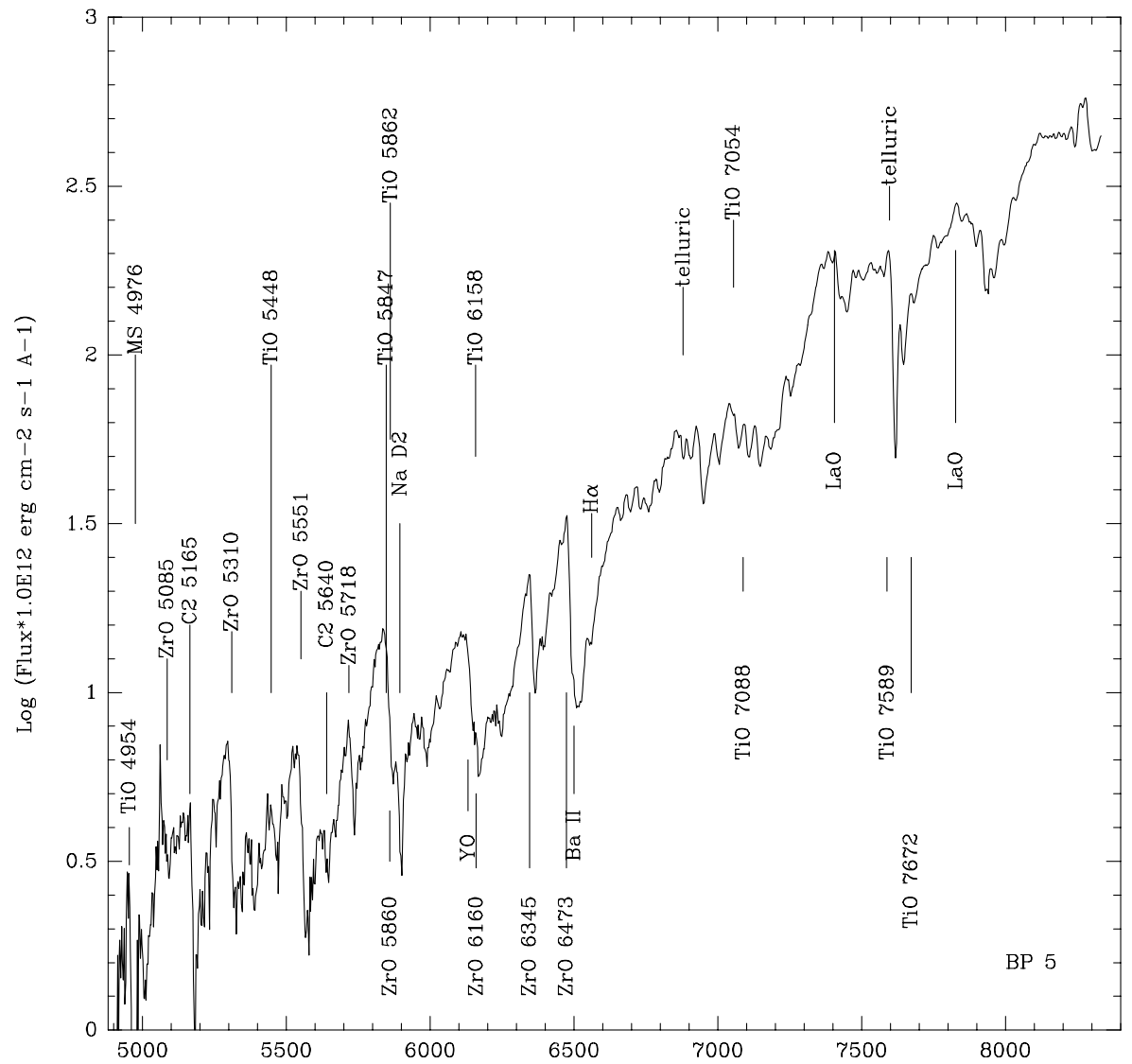

Fig. 8. The flux calibrated spectrum of BP 5 in the $4800-8400 \AA$ range. Lines and bands used for spectral classification are shown 

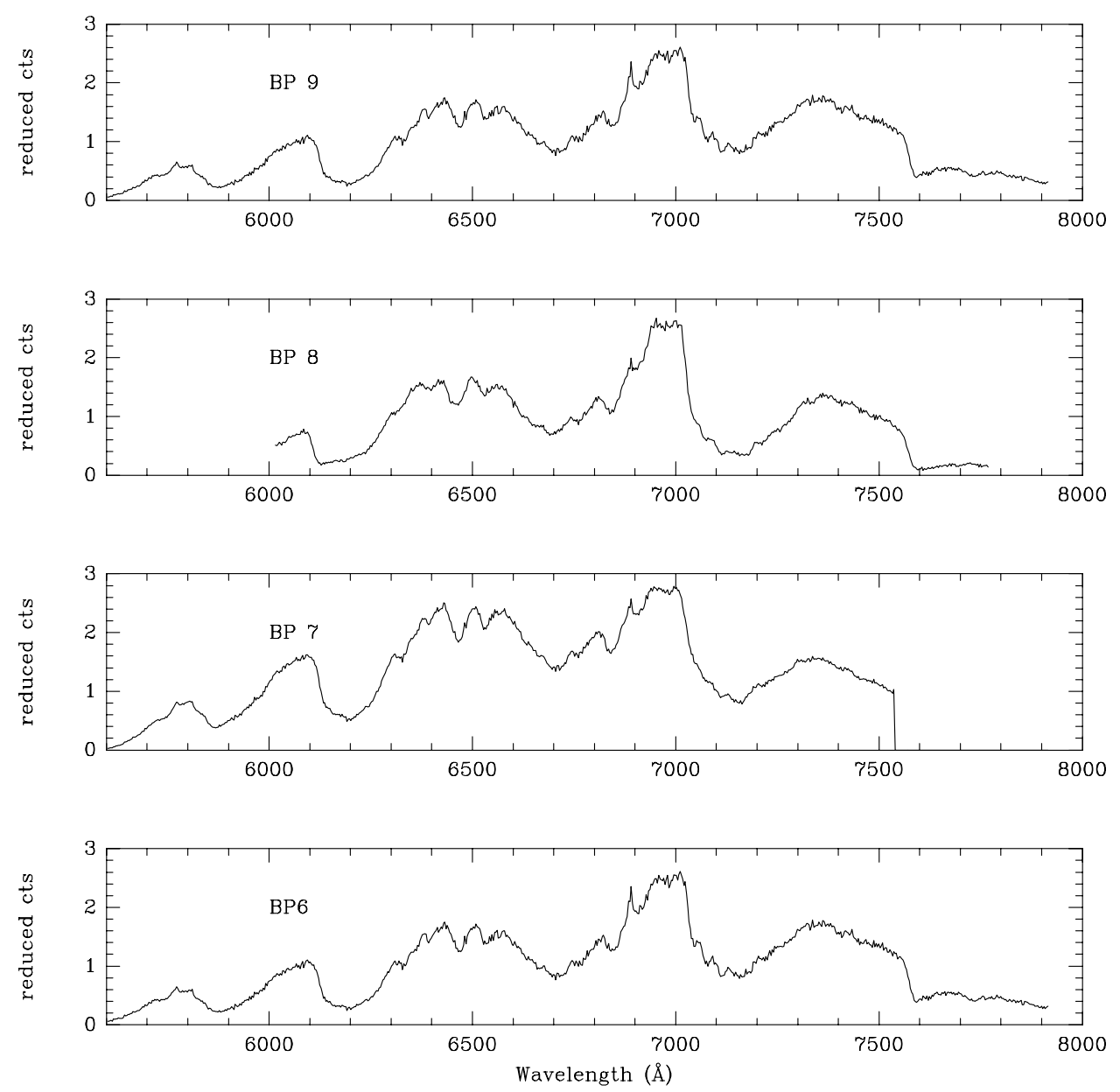

Fig. 9. The low resolution spectra of BP 6, BP 7, BP 8 and BP 9 in the 5600-8000 $\AA$ range. Fluxes are only corrected for instrumental effects. Some spectra are cut because of overlapping in our slitless spectroscopic images

\section{References}

Barnbaum, C., Stone, R. P. S., \& Keenan, P. C. 1996, ApJS, 105,419

Battinelli, P., \& Capuzzo-Dolcetta, R. 1991, MNRAS, 249, 76

Caloi, V., \& Cassatella, A. 1998, A\&A, 330, 492

Fabregat, J., \& Reglero, V. 1990, MNRAS, 247, 414

Fabregat, J., \& Torrejon, J. M. 1999, Proc. of the IAU Colloq. 175 , The Be phenomenon in early type stars, Alicante 28th June -2nd July ASP Conf. Ser., in press

Jaschek, C., \& Jaschek, M. 1987, The classification of stars (Cambridge Univ. Press)

Jacoby, G. H., Hunter, D. A., \& Christian, C. A. 1984, ApJS, 56,257

Jorgensen, U. G. 1985, Ap\&SS, 114, 181

van der Hucht, K. A., Conti, P. S., Lundström, I., \& Stenholm, B. 1981, Space Science Rev., 28, 227

Keenan, P. C., \& Boeshaar, P. C. 1980, ApJS, 43, 79

Kirkpatrick, J. D., Henry, T. J., \& Mc Carthy, D. W. Jr. 1991, ApJS, 77, 414

Kurucz, R. L. 1979, ApJS, 40, 1

Lennon, D. J., Dufton, P. L., \& Fitzsimmons, A. 1992, A\&AS, 94,569
MacConnell, D. J., \& Coyne, G. V. 1983, Vatican Obs. Publ., 2,63

Maeder, A. 1996, Proc. of the 33rd Liege Astrophysical Colloquium on The WR stars in the framework of stellar evolution (Liege University press) 39-56

Massey, P., Johnson, J., \& DeGioia-Eastwood, K. 1995, ApJ, 454,151

Merighi, R., Mignoli, M., Ciattaglia, C., et al. 1994, BFOSC User's Manual, RT 09-1994-05, Bologna Astronomical Observatory

Polcaro, V. F., \& Viotti, R. 1997, Proc. of the VII ADASS Conferences, ASP Conf. Ser., 145, 78

Schaller, G., Schaerer, D., Meynet, G., \& Maeder, A. 1992, A\&AS, 96, 269

Schmidt-Kaler, Th. 1982, in Landolt, Bornstein: Numerical Data and Functional Relationship in Science and Technology, vol. 2b, ed. K. Schaifers, \& H. H. Voigt (Springer, Berlin)

Steele, I. A., Neguerela, I., \& Clark, J. S. 1999, A\&AS, 137, 147

Zombeck, M. V. 1990, Handbook of Astronomy and Astrophysics, Second Edition (Cambridge University Press, Cambridge) 